\title{
Monitoring D-region Variability from Lightning Measurements
}

\author{
Fernando Simöes ${ }^{1}$, Jean-Jacques Berthelier ${ }^{2}$, Robert Pfaff ${ }^{l}$, Dieter Bilitza ${ }^{3,4}$, and Jeffrey Klenzing ${ }^{l}$ \\ ${ }^{1}$ NASA/GSFC Heliophysics Science Division, Space Weather Laboratory, 8800 Greenbelt Road, Greenbelt, Maryland, \\ 20771, USA (fernando.a.simoes@nasa.gov; robert.f.pfaff@nasa.gov; jeffrey.klenzing@nasa.gov) \\ ${ }^{2}$ LATMOS/IPSL, UPMC, 4 Place Jussieu, 75005 Paris, France (jean-jacques.berthelier@latmos.ipsl.fr) \\ ${ }^{3}$ NASA/GSFC Heliophysics Science Division, Heliospheric Physics Laboratory, 8800 Greenbelt Road, Greenbelt, \\ Maryland, 20771, USA (dieter.bilitza-1@nasa.gov) \\ ${ }^{4}$ George Mason University, Space Weather Laboratory, Fairfax, Virginia, USA (dbilitza@gmu.edu)
}

\begin{abstract}
In situ measurements of ionospheric D-region characteristics are somewhat scarce and rely mostly on sounding rockets. Remote sensing techniques employing Very Low Frequency (VLF) transmitters can provide electron density estimates from subionospheric wave propagation modeling. Here we discuss how lightning waveform measurements, namely sferics and tweeks, can be used for monitoring the D-region variability and day-night transition, and for local electron density estimates. A brief comparison among D-region aeronomy models is also presented.
\end{abstract}

\section{Introduction}

The ionospheric D-region is extremely difficult to study not only because the chemistry is complex but in situ measurements are scarce and sometimes unreliable. Unlike the regions above where only positive ions and electrons are important, negative ions also play a key role in the D-region. Additionally, presence of metallic particles, provided by meteoritic bombardment, and heavy cluster ions produce intricate chemical reactions in the mesosphere. On the other hand, the D-region altitude range is too low for satellites and too high for atmospheric balloons. Except for sounding rocket in situ measurements made mostly during day, the D-region is usually investigated through propagation techniques that effectively provide data at much larger scales, employing satellites, rockets, and ground-based transmitters. An alternative to Very Low Frequency (VLF) ground-based transmitters is using lightning signatures, namely sferics and tweeks, to study the D-region variability through subionospheric wave propagation modeling. In this work we report VLF electric field measurements made onboard a stratospheric balloon during the African Monsoon Multidisciplinary Analyses (AMMA) SCOUT campaign [1,2]. The balloon flight lasted about $3 \mathrm{~h}$, encompassing the time of sunset, thus contributing to assessing the variation of the propagation conditions due to the D-region electron density changing. After a brief description of the experiment and atmospheric conditions of the flight, we provide the results of an analysis of several hundred impulsive electromagnetic events for which the transverse resonance mode of the surface-ionosphere waveguide is positively identified. These observations are in fair agreement with a wave propagation model that considers an electron density distribution near dusk derived from the International Reference Ionosphere (IRI) model [3], considering three representations of the chemical processes.

\section{Instrument Description and Balloon Measurements}

The experiment took place on 7 August 2006 in Niamey, Niger (geographic coordinates 13.5N, 2E), and the balloon was launched at TO $=1645$ UT. With an ascent velocity of $\sim 5 \mathrm{~ms}^{-1}$, the balloon reached the ceiling altitude of 23 $\mathrm{km}$ at 1802 UT, traveling $\sim 200 \mathrm{~km}$ almost exactly westward until the end of the flight. During the ceiling period, the balloon altitude decreased slowly down to about $20 \mathrm{~km}$ after $1845 \mathrm{UT}$; the last measurements were recorded at $19 \mathrm{~km}$. The sunset at the balloon position occurred at 1843 UT (TB, $118 \mathrm{~min}$ from T0); the sunset at the position of the strongest local convective system $(-400 \mathrm{~km}$ east of the balloon final position) and in the D-region above occurred 93 
(TS) and $130 \mathrm{~min}$ (TD) from T0, respectively. Images from the AMMA campaign meteorological radar showed active, though variable, convective systems about $\sim 100 \mathrm{~km}$ east and south of Niamey [2]. Numerous lightning and strong electric field transients were observed.

The HV-AIRS (High Voltage - Atmospheric Impact of Radiations and Sprites) electric field experiment was part of a multiple instrument flight chain that included other sensors devoted to observations of aerosols and atmospheric composition. It measures the atmospheric electric field from $\mathrm{DC}$ to $\sim 4 \mathrm{kHz}$ employing the double probe technique, which has been widely used onboard balloons and satellites to measure electric fields. The effective length of the antenna is about $40 \mathrm{~cm}$ and the DC and AC components of the electric field are measured in two independent channels. The $\mathrm{AC}$ signals are measured in the frequency range from $4 \mathrm{~Hz}$ to $4 \mathrm{kHz}$, the noise level is $30 \mathrm{mVm}^{-1} \mathrm{~Hz}^{-1 / 2}$, and multiple gain settings allow for measuring electric fields up to $\pm 2.5 \mathrm{Vm}^{-1}$. Although aiming a proof-of-concept for planetary missions - consequently, the physical parameters are very much limited - instrument sensitivity and sampling are sufficient for sferics and tweeks detection. The HV-AIRS gondola also includes simple optical detectors to identify lightning as far as $\sim 300 \mathrm{~km}$ after sunset and a GPS receiver. Figure 1 illustrates typical sferics and tweek waveforms recorded during the flight. Most waveforms are detected during nighttime because propagation conditions are more favorable, i.e., the ionosphere is sharper and offers better reflection conditions for VLF subionospheric wave propagation.
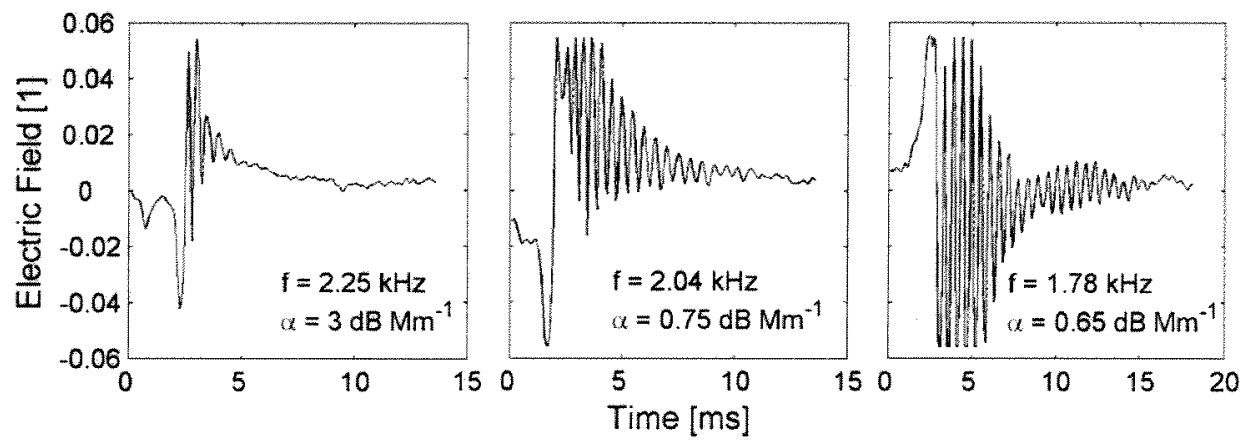

Figure 1: Typical (left) day and (middle) night sferics recorded by HV-AIRS onboard the stratospheric balloon at altitude of about $20 \mathrm{~km}$. (right) Distinctive tweek recorded during nighttime at $19 \mathrm{~km}$ (adapted from [6]).

\section{Numerical Modeling}

To understand the balloon data, we have developed a wave propagation finite element model that benefits from algorithms previously used to study wave propagation on Earth and other planetary environments [4, 5]. The model is run in the transient and harmonic wave propagation modes to compute the electric fields in the surface-ionosphere waveguide. For the transient mode, we consider a vertical current element with a typical lightning current profile. The stationary mode computes the electric field spectrum of the waveguide in the range $0-10 \mathrm{kHz}$, considering a vertical Hertz dipole. Below $60 \mathrm{~km}$, a scalar conductivity profile is used. Above $60 \mathrm{~km}$, we use the parallel, Pedersen, and Hall components of the conductivity tensor, considering the Appleton-Hartree equation which describes the refractive index in cold magnetized plasma [6]. The electron density is derived from the International Reference Ionosphere (IRI) model, including the three parameterizations of the D-region: (i) default (IRI-2007) - IRI-1 [7], (ii) Friedrich (FIRI) IRI-2 [8,9], and Danilov - IRI-3 [10]. Figure 2 shows transient signals at the balloon ceiling altitude computed with the transient method. The discharge starts at $t=1 \mathrm{~ms}$ and the electric field is computed in several locations, i.e., for various angular separations between the radiating source and the balloon. The frequency of the waveform is $\sim 2 \mathrm{kHz}$ and the propagation delay increases with angular separation. It is worth mentioning that the quasi-static component decreases with distance and a clean sinusoidal waveform is observed. Although lightning stroke accurate characterization considering intensity, duration, distance, and direction requires triangulation and receiver synchronization, it is possible to estimate the source distance from waveform morphology, i.e., shape, attenuation, and quasi-static component contribution. Comparison between the balloon data and model waveforms suggests that about $75 \%$ of the recorded lightning occurred no farther than $350 \mathrm{~km}$, i.e., $3^{\circ}$ of angular separation, which is significant for the day-night transition accurate detection. 

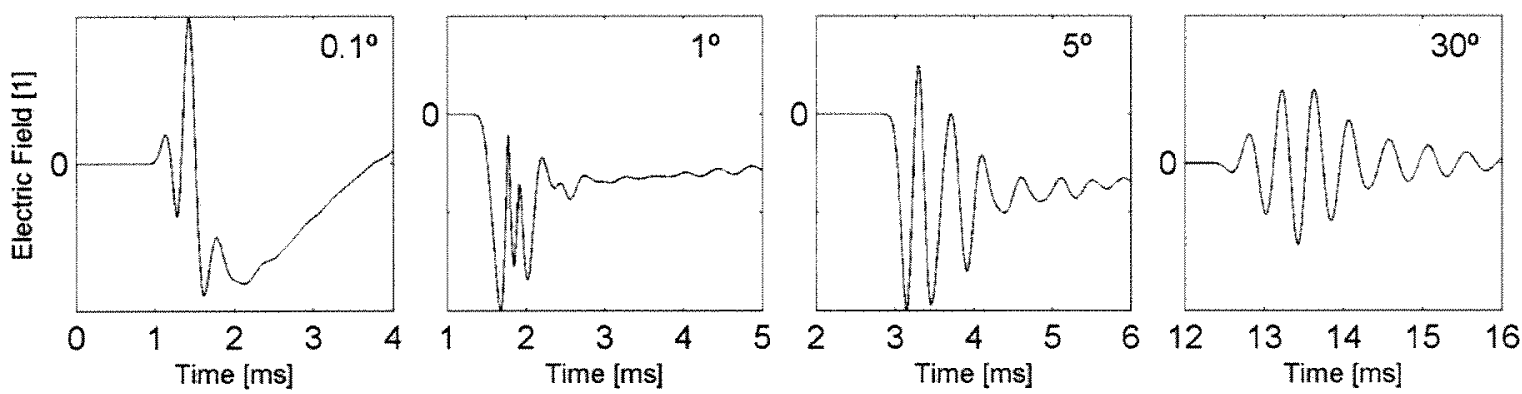

Figure 2: Waveforms of a lightning discharge calculated with the transient mode algorithm for several angular separations from the source.

\section{Results and Discussion}

From the few thousand impulsive events recorded by the $\mathrm{AC}$ channel we are able to retrieve frequency information from about 700 sferics and a few tweeks. Three approaches are tested to compute the waveform mean period, namely the method of least squares applied to damped sinusoidal functions, Fourier analysis, and crest-crest period counting. From typical events shown in Figure 1, we can see that the frequency, $f$, and wave attenuation, $\alpha$, are lower for nighttime propagation. These results are consistent with wave reflection in a sharper layer at higher altitude, in line with IRI predictions. Figure 3 presents the frequencies derived from sferics and tweeks waveforms. Although the rate of lightning strokes is roughly constant during flight, the events from which frequency calculation is reliable are concentrated after sunset, an indication of more favorable propagation conditions during nighttime. The histogram on the right-hand-side provides the number of events as a function of frequency, showing a broad peak about $2 \mathrm{kHz}$.

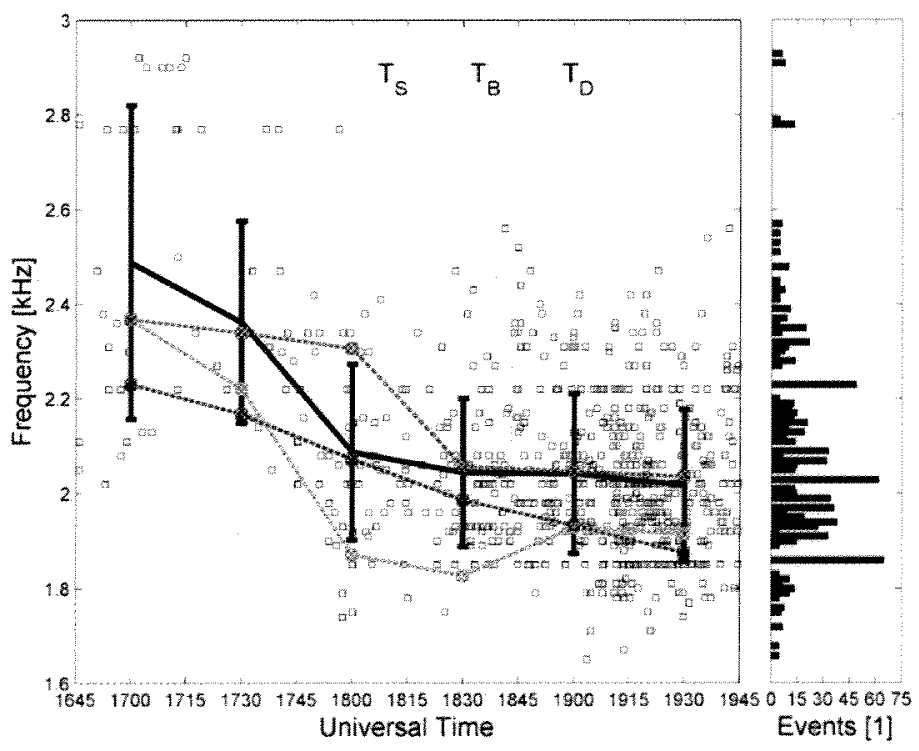

Figure 3: Comparison between frequencies derived from sferics and tweeks and our numerical model. (left) Squares correspond to the frequency of individual impulsive events; the solid line and error bars represent the mean value and standard deviation calculated over periods of $30 \mathrm{~min}$. The colored lines are numerical results using IRI electron density distributions, considering (blue) IRI-1, (green) IRI-2, and (red) IRI-3 model options for the IRI D-region. The labels $T_{S}$, $T_{B}$, and $T_{D}$ represent the sunset time at the strongest convective cell, balloon position, and the D-region above the convective cell, respectively. (right) Histogram of impulsive events as function of the mean frequency measured during the flight. 
During the solar eclipse of 7 March 1970, Reeve and Rycroft [11] characterize tweeks resulting from nighttime propagation conditions to determine the height of the ionospheric reflector, and are able to infer the change of the effective height of the ionosphere during the eclipse. They obtain 69 and $76 \mathrm{~km}$ at the start/finish and maximum totality of the eclipse. A previous study of the present data shows that the day-night ionospheric depletion occurring in the altitude range from 73 to $82 \mathrm{~km}$ before and after sunset, respectively [6]. These altitudes are somewhat lower that those suggested by other studies [cf. 6 and references therein] because the present data are recorded close to sunset, when the ionosphere has not yet reached an ionization minimum. Several conclusions can be drawn from Figure 3: (i) IRI-2 and IRI-3 show sharper day-night transitions than that of IRI-1, in line with the balloon measurements; (ii) the frequency range derived from the IRI-3 model shows a better agreement with data despite a 30 min offset; (iii) Although consistently underestimating the average frequency by $\sim 0.15 \mathrm{kHz}$, the IRI-2 model reasonably matches the profile. Under the assumption these results are representative and can be generalized to other latitudes and longitudes, a more elaborate model would perhaps combine IRI-2 electron density magnitude with IRI-3 electron density gradients.

In this work we show that lightning is a valuable tool to study the day-night transition of the ionospheric Dregion. A straightforward comparison among three models of the D-region (IRI-1, IRI-2, and IRI-3) unveils their relative accuracy for deriving electron density distributions. Careful monitoring of the ionospheric day-night variation may eventually provide additional information for investigating the chemical processes taking place in the D-region.

\section{Acknowledgments}

FS and JK are supported by an appointment to the NASA Postdoctoral Program at the Goddard Space Flight Center, administered by Oak Ridge Associated Universities through a contract with NASA. This work benefited from financial support by CNES and was made possible thanks to the AMMA SCOUT project. Fruitful discussions with AMMA team members are acknowledged.

\section{References}

[1] F. Cairo et al., "An introduction to the SCOUT-AMMA stratospheric aircraft, balloons and sondes campaign in West Africa, August 2006: rationale and roadmap", Atmos. Chem. Phys. 10, 2237-2256, 2010.

[2] AMMA data center at http://aoc.amma-international.org.

[3] D. Bilitza and B. Reinisch, "International Reference Ionosphere 2007: Improvements and new parameters," $A d v$. Space Res. 42, 599-609, doi:10.1016/j.asr.2007.07.048, 2008.

[4] F. Simões et al., "A new numerical model for the simulation of ELF wave propagation and the computation of eigenmodes in the atmosphere of Titan: Did Huygens observe any Schumann resonance?," Planet. Space Sci. 55 19781989, 2007, doi:10.1016/j.pss.2007.04.016, 2007.

[5] F. Simões et al., "The Schumann resonance: A tool for exploring the atmospheric environment and the subsurface of the planets and their satellites," Icarus 194, 30-41, doi:10.1016/j.icarus.2007.09.020, 2008.

[6] F. Simões, J.-J. Berthelier, M. Godefroy, and S. Yahi., "Observation and modeling of the Earth-ionosphere cavity electromagnetic transverse resonance and variation of the D-region electron density near sunset," Geophys. Res. Lett. 36, L14816, doi:10.1029/2009GL039286, 2009.

[7] D. Bilitza, "Electron density in the D-region as given by the IRI," in International Reference Ionosphere - IRI 79, edited by V. Lincoln, R. Conkright, and K. Rawer, Rep. UAG-82, pp. 7-10, World Data Cent. A for Sol.-Terr. Phys., Boulder, Co., Nov. 1981.

[8] M. Friedrich and K.M. Torkar, "An empirical model of the nonauroral D Region," Radio Sci. 27, 945-953, 1992.

[9] M. Friedrich and K.M. Torkar, "FIRI: A semiempirical model of the lower ionosphere," J. Geophy. Res. 106, 21409-21418, 2001.

[10] A. Danilov, A. Rodevich, and N. Smirnova, "Problems with incorporating a new D-region model into the IRI," Adv. Space Res. 15, 165-169, 1995.

[11] C.D. Reeve and M.J. Rycroft, "Eclipsed lower ionosphere as investigated by natural very low frequency radio signals", J. Atmos. Terr. Phy. 34, 667-672, 1972. 